\title{
Identification of the non-elastic material parameters by inverse modelling: A stress based approximation
}

\author{
I. Vasilakos ${ }^{1, a}$, J. Gu ${ }^{1}$ and H. Sol ${ }^{1}$ \\ ${ }^{1}$ Faculty of Applied Sciences, Department of Mechanics of Materials and Constructions, Vrije \\ Universiteit Brussel, Pleinlaan 2, B-1050 Brussels, Belgium
}

\begin{abstract}
Inverse methods are a modern alternative technique to traditional standard testing for the identification of material parameters. The material parameters in the numerical model are the unknown parameters which will be aimed to identify. In this paper an alternative iterative strategy for material identification is proposed. The concept of the proposed strategy is based on the material behavior under an applied load which is described by the stress-strain curve. The material parameters can be identified based on observation strain field and numerically stress field.
\end{abstract}

\section{Introduction}

New advances in solid Mechanics are linked to close and adequate interactions between experiments, modelling and simulations. In particular, the finite element method has become a classical means used in various industries as a design tool. This progress opens the possibility to design reliable and sustainable structures up to failure. However, it requires that numerical tools are accurate and validated, and that the used models are able to capture the most meaningful physical aspects of the material behaviour. [1]

In order to numerically simulate the mechanical behaviour of materials under extreme strain conditions it is necessary to develop appropriate constitutive models. These models contain a set of unknown parameters which have to be identified from experimental processes. Thanks to the revolution of the I.T. technology numerical models could be used to solve miscellaneous problems. The most powerful method is the finite-element method, which yields approximate solutions to differential equations that model problem arising in physics and engineering. Finite element analysis requires accurate material models for the realistic description of the material behaviour.

Inverse methods compare experimental observations with numerical simulations. The material parameters in the numerical model are the unknown parameters which will be aimed to identify. The inverse modelling can tune the different material parameters in a single experimental setup. In the past several attempts have been made to identify elastic parameters based on inverse modelling of different experimental set-ups. Among others, some researchers used uniaxial tensile tests on a perforated beam-like specimen [2], others have used a transversely loaded rectangular plate [3], a

\footnotetext{
a e-mail : ivasilak@vub.ac.be
}

This is an Open Access article distributed under the terms of the Creative Commons Attribution-Noncommercial License 3.0, which permits unrestricted use, distribution, and reproduction in any noncommercial medium, provided the original work is properly cited. 
circular disk under diametrical compression [4], a T-shaped specimen under tensile loading [5] or the displacement information around a hole in a bi-axially loaded plate [6,7].

In the current study, a stress based strategy is used to identify the non-elastic material parameters. A simulated experiment is used to illustrate the principle of the method.

\section{Principle of the stress based strategy}

The inverse method described in this work can actually be narrowed to parameter estimation, as the only item of interest to this study is the determination of the constitutive model parameters. The values of these parameters cannot be derived immediately from a traditional experiment. A numerical analysis is necessary to simulate the actual experiment. However, this requires that initial values of the material parameters are known. The identification problem can then be formulated as a convergence problem, in which the material elasto-plastic behaviour ( $\sigma-\varepsilon$ hardening curve) is updated until it converges to a final form. From the final obtained $\sigma-\varepsilon$ hardening curve, material parameters of a chosen material model can be extracted.

Figure 1 illustrates the general concept of a stress based inverse method for material identification. The dashed line in the flow chart corresponds to the iterative material behaviour process. The described inverse method is based on the concept that the elasto-plastic material behaviour of a metal can be described by the strain-stress $(\sigma-\varepsilon)$ hardening curve. This means that for the FE simulation the material parameters are not required since the strain-stress hardening curve is known and contains all necessary information. The material identification process can be described using the following steps:

- From the experiment the strain field is measured.

- From the FE-model with an initially assumed elasto-plastic material behaviour, the stress field in the test specimen is computed

- Composition of an intermediate strain-stress curve based on the experimentally measured equivalent strain field and the numerically computed equivalent stress field.

- Implementation of the first intermediate curve as updated material model in the FEM.

- New iteration steps until convergence is achieved

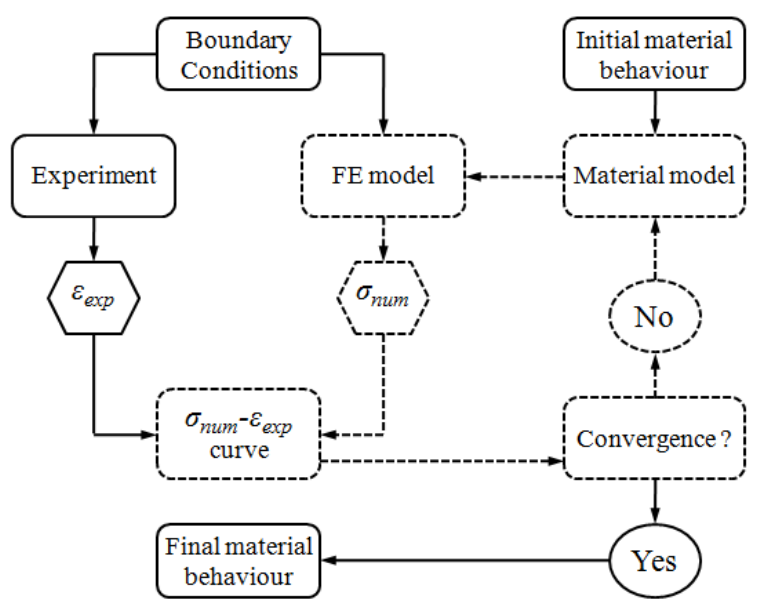

Fig. 1. Flowchart of the inverse method for material parameter identification. The square dot line indicates the iterative part of the method. 


\subsection{Non-elastic identification}

The elasto-plastic material model on which this work is focused, is based on an additive decomposition of the total strain increments into elastic and plastic parts. The hardening of the material is assumed to be isotropic following the Von Mises yield criterion and it is modelled by the Nadai relation.

\subsubsection{The Von Mises Yield criterion}

The Von Mises yield criterion suggests that yielding in materials begins when the second deviatoric stress invariant $J_{2}$ reaches a critical value $K$. Because the Von Mises criterion [8] is independent from the first stress invariant, $J_{l}$, it is applicable for the analysis of plastic deformation for ductile materials such as metals. The criterion can be expressed in terms of stress tensor components as:

$$
\left(\sigma_{11}-\sigma_{22}\right)^{2}+\left(\sigma_{22}-\sigma_{33}\right)^{2}+\left(\sigma_{33}-\sigma_{11}\right)^{2}+6\left(\sigma_{23}^{2}+\sigma_{31}^{2}+\sigma_{12}^{2}\right)^{2}=2 \sigma_{e q}^{\text {Mises }}
$$

For plane stress conditions where the plane of the sheet material is parallel to (1,2)-plane the Von Mises criterion is represented by:

$$
\sigma_{11}^{2}+\sigma_{22}^{2}-2 \sigma_{11} \sigma_{22}+6 \sigma_{12}^{2}=2 \sigma_{e q}^{\text {Mises }}
$$

The equivalent strain according to the Von Mises yield criterion is expressed as:

$$
\varepsilon_{11}^{2}+\varepsilon_{22}^{2}+2 \sigma_{12}^{2}=\frac{3}{2} \varepsilon_{e q}^{\text {Mises }}
$$

The most general formulation for the equivalent plastic strain rate is found by considering the Euclidean norm of the plastic strain rate tensor. The value of the total equivalent plastic strain can then be computed by integration of the equivalent plastic strain rate over the load history:

$$
\varepsilon_{e q}^{p l}=\int_{0}^{t} \dot{\varepsilon}_{e q}^{p l} d t
$$

\subsubsection{The Ludwik-Nadai relation for isotropic hardening}

Strain hardening is the strengthening of a material subjected to plastic deformation. As the material becomes increasingly saturated with new dislocations, new dislocations are prevented from nucleating (a resistance to dislocation-formation develops). This resistance to dislocation-formation manifest itself as a resistance to plastic deformation; hence, the observed strengthening. The mathematical description of the strain hardening, is called hardening law.

A frequently used strain hardening model is given by the Ludwik-Nadai or Nadai relation . It allows a good approximation of a large part of the true stress-train curve:

$$
\sigma_{e q}^{p l}=K\left(\varepsilon_{e q}^{p l}\right)^{n}
$$

With $K$ the deformation resistance and $n$ the hardening exponent. Isotropic hardening is assumed if loading is inversed after a first monotonic loading (up to $\sigma_{l}$ ), the second yield point is symmetrical with respect to the maximum stress in monotonic loading $\left(-\sigma_{l}\right)$. 


\section{Numerical evaluation of the inverse method}

For the identification of the elasto-plastic parameters, a bi-axial tensile test was used. A numerical experiment was used to simulate a virtual experiment. The presence of the holes in the specimen ensured the generation of a heterogeneous field of strains and stresses. Figure 2 illustrates the geometry of the specimen and the applied boundary conditions. The FE-simulations are based on linear iso-parametric triangular elements of the Lagrange type. As the thickness of the specimen (1 $\mathrm{mm}$ ) is rather small compared to its length and width and the applied tensile load is in the plane of the specimen, plane stress conditions can be assumed.

The first assumption concerning the used material model is that it exhibits strain rate independent elasto-plastic behaviour. Furthermore the elastic part of the deformation is assumed to be linear with isotropic material behaviour. This implies that the stress rate is, at any moment in time, related to the elastic strain rate according to generalized Hook's law:

$$
\dot{\boldsymbol{\sigma}}=E: \dot{\boldsymbol{\varepsilon}}_{\mathrm{el}}
$$

The non-elastic behaviour of the material is isotropic which means that the plastic deformation is independent of the orientation of the coordinate axes in which the stresses are expressed. Von Misses criterion is valid with these assumptions. The constitutive law used in the FE-formulation is based on the decomposition of the total strain into a reversible elastic part $\varepsilon_{e l}$ and an irreversible plastic part $\varepsilon_{p l}$. In rate form can be expressed as:

$$
\dot{\boldsymbol{\varepsilon}}=\dot{\boldsymbol{\varepsilon}}_{\mathrm{el}}+\dot{\boldsymbol{\varepsilon}}_{\mathrm{pl}}
$$

In the present study a Ludwik-Nadai relation of hardening model is assumed. The elastic parameters are assumed to be known. The material parameters which have to be identified are hence $K$ and $n$.
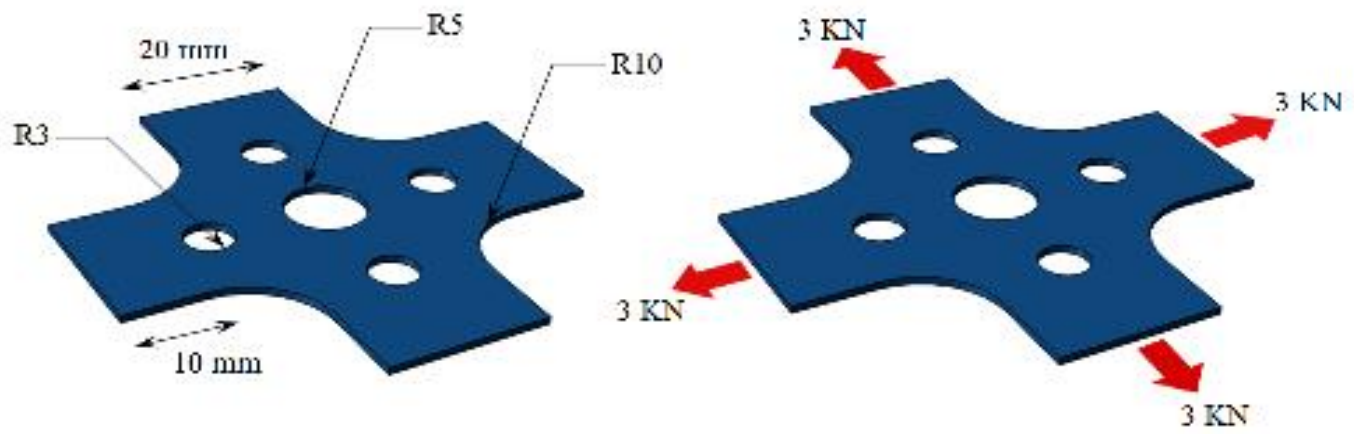

Fig. 2. Geometry and boundary conditions of the cruciform specimen subjected to bi-axial loading.

The values of the 'real' material parameters which characterize the behaviour (elastic and nonelastic) of the material are given in table 1 .

Table 1. The values of the parameters used for the simulated experiment

\begin{tabular}{|c|c|c|c|c|c|}
\hline & $\begin{array}{c}\mathrm{E} \\
(\mathrm{GPa})\end{array}$ & $\begin{array}{c}\mathrm{v} \\
(-)\end{array}$ & $\begin{array}{c}\sigma_{0} \\
(\mathrm{MPa})\end{array}$ & $\begin{array}{c}\mathrm{K} \\
(\mathrm{MPa})\end{array}$ & $\begin{array}{c}\mathrm{n} \\
(-)\end{array}$ \\
\hline Real values & 70 & 0.32 & 50 & 180 & 0.2 \\
\hline
\end{tabular}

The resulting deformation, computed in different integration points, is given by the equivalent strain which is considered to be 'experimentally' obtained. The objective is to identify the values of the material parameters of the Ludwik-Nadai relation used for the virtual experiment. The selected set of initial values, used to initialize the identification procedure, is given in table 2 . 
Table 2. The initial values of the parameters used for the identification process

\begin{tabular}{|c|c|c|c|c|c|}
\hline & $\begin{array}{c}\mathrm{E} \\
(\mathrm{GPa})\end{array}$ & $\begin{array}{c}\mathrm{v} \\
(-)\end{array}$ & $\begin{array}{c}\sigma_{0} \\
(\mathrm{MPa})\end{array}$ & $\begin{array}{c}\mathrm{K} \\
(\mathrm{MPa})\end{array}$ & $\begin{array}{c}\mathrm{n} \\
(-)\end{array}$ \\
\hline Real values & 70 & 0.32 & 50 & 250 & 0.25 \\
\hline
\end{tabular}

\section{Results and discussion}

Two different simulations were completed for the identification of the material parameters. The first simulation represented the simulated experiment. The second simulation according to the stress based strategy was corresponding to the initial FE-model. Figure 3 illustrates the distribution of the Von Misses stress and the equivalent strain in terms of coloured contours. The holes generate a heterogeneous stress and strain field.

According to the principle of the inverse method, the strain field was measured from the simulated experiment and the stress field was taken from the initial FE-model. The intermediate curve was composed by the 'experimental' strains and the numerical stresses. This curve was implemented as material model to the FE simulation. Figure 4 illustrates the non-elastic behaviour of the material in terms of equivalent true strain and stress of the initial FE-model, the simulated experiment and the $1^{\text {st }}$ intermediate curve.

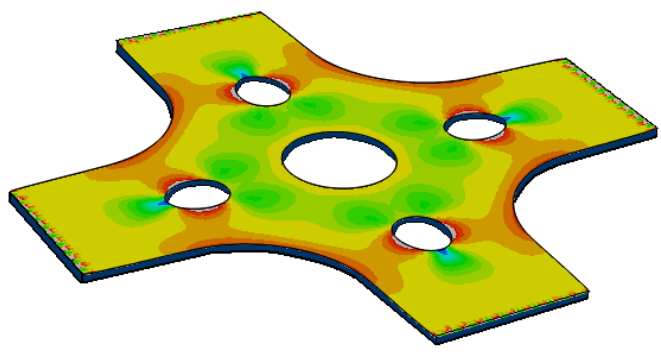

(a)

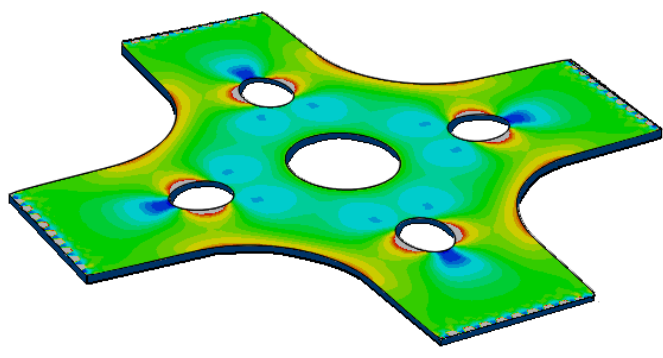

(b)

Fig. 3. Distribution of the Von Mises (a) stress and (b) strain field in the cruciform specimen. The holes generate a heterogeneous field

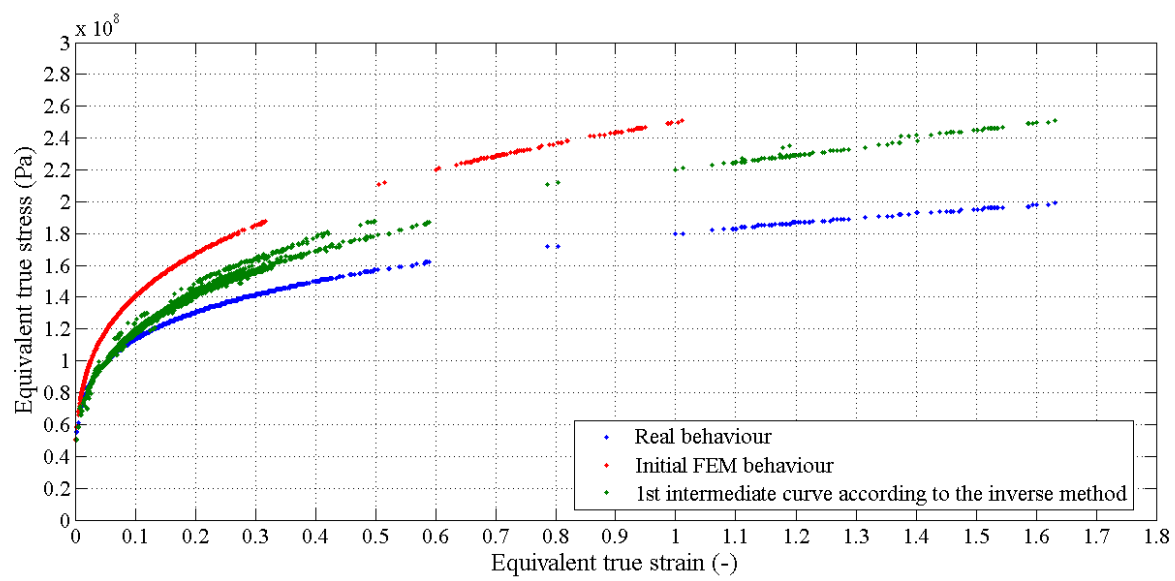

Fig. 4. Composition of the $1^{\text {st }}$ intermediate strain-stress curve according to the initial stress field and the experimental strain field. 
Since the $1^{\text {st }}$ intermediate strain-stress curve is composed, the parameters of the Ludwik-Nadai relation can be exported through an algorithm. This algorithm curve fits the Ludwik-Nadaigh expression through the minimization of the residuals. The tuned expression of the hardening behaviour can then be implemented in the FE-model as new non-elastic material model. This iterative process is repeated until a convergence to the 'real' non-elastic behaviour is achieved. Figure 5 illustrates the convergence of the non-elastic behaviour of the cruciform specimen after seven iterations.

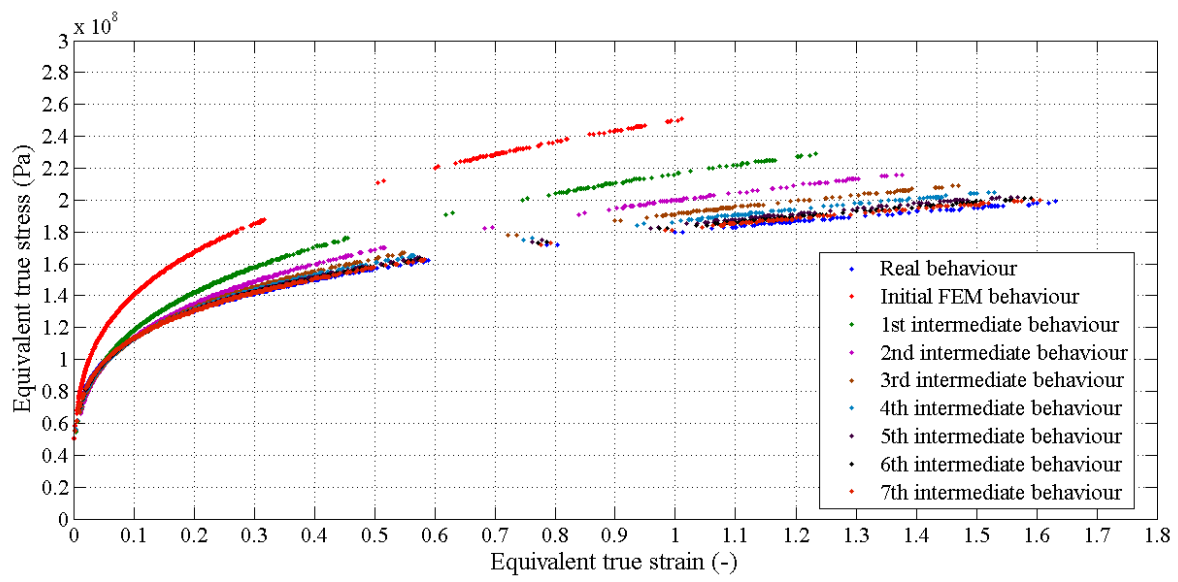

Fig. 4. Convergence of the FEM non-elastic behaviour to the real behaviour after seven iterations.

Table 3 presents the obtained non-elastic parameters according to the Ludwik-Nadai relation. In figure 5 is illustrated the convergence of the non-elastic parameters after seven iterations.

Table 3. The initial values of the parameters used for the identification process

\begin{tabular}{|c|c|c|c|c|}
\hline & $\begin{array}{c}\mathrm{K} \\
(\mathrm{MPa})\end{array}$ & $\begin{array}{c}\text { r. error K } \\
(\%)\end{array}$ & $\begin{array}{c}\mathrm{n} \\
(-)\end{array}$ & $\begin{array}{c}\text { r. error } \mathrm{n} \\
(\%)\end{array}$ \\
\hline Initial values & 250.00 & 38.88 & 0.250 & 25.0 \\
\hline $1^{\text {st }}$ iteration & 216.45 & 20.25 & 0.262 & 31.0 \\
\hline $2^{\text {nd }}$ iteration & 199.87 & 11.03 & 0.245 & 22.5 \\
\hline $3^{\text {rd }}$ iteration & 191.36 & 6.31 & 0.229 & 14.5 \\
\hline $4^{\text {th }}$ iteration & 186.70 & 3.72 & 0.218 & 9.0 \\
\hline $5^{\text {th }}$ iteration & 184.06 & 2.61 & 0.211 & 5.5 \\
\hline $6^{\text {th }}$ iteration & 182.51 & 1.39 & 0.207 & 3.5 \\
\hline $7^{\text {th }}$ iteration & 180.62 & 0.34 & 0.201 & 0.5 \\
\hline
\end{tabular}
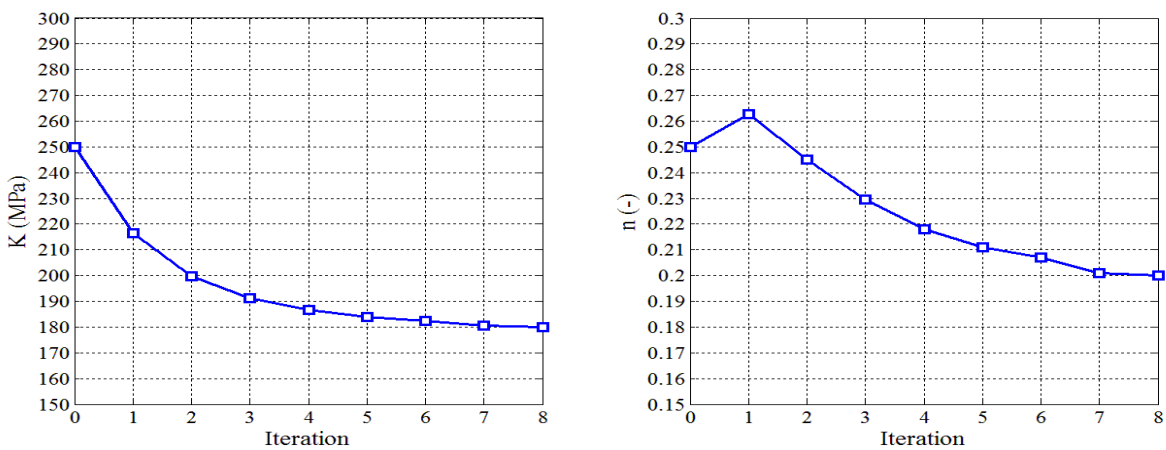

Fig. 4. Convergence of the non-elastic parameters $K$ and $n$ after seven iterations. 


\section{Conclusions}

This study was focused to the identification of the non-linear parameters of a cruciform specimen subjected to bi-axial loading, through an inverse method, more specifically the identification of the parameters of the Ludwik-Nadai hardening law.

The inverse method which was used during this study, is taking into acount the generated stress field from the FE simulation. As a result the material identification can be defined as a convergence problem. The main advantage of this method as compared with many other inverse methods is that it does not require the minimization of a cost function. This generates the prospective that the computation time can be decreased and the calculation of the Jacobian matrix during the parameters updating could be avoided.

\section{Acknowledgments}

The authors would like to acknowledge the financial support from the Flemish Institute of Fundamental Sciences (FWO).

\section{References}

1. S. Roux, F. Hild, Experimental mechanics, 48, 4 (2008)

2. J. Molomard, Le Riche, A. Vautrin, J.R. Lee, Experimental mechanics, 45, 5 (2005)

3. W. Wang, T. Kam, Composite Structures 50, (2000)

4. Z. Wang, J. Cardenas-Garcia, B. Han, Experimental mechanics, 45, 1 (2005)

5. M. Grediac, F. Pierron, Y. Surrel, Experimental Mechanics, 39, (1999)

6. J.Cardenas-Garcia, Mechanics Research Communications, 27, 1, (2000)

7. D. Lecompte, A. Smits, H. Sol, J. Vantomme, D. Van Hemerlrijck, International Journal of Solids and Structures, 44, (2006)

8. R. Hill, The mathematical theory of plasticity, (1950) 